\title{
Mycelial Growth and Branching of Streptomyces coelicolor A3(2) on Solid Medium
}

\author{
By EUNICE J. ALLAN* AND J. I. PROSSER \\ Department of Microbiology, University of Aberdeen, Marischal College, \\ Aberdeen $A B 9$ 1AS, U.K.
}

(Received 10 January 1983; revised 14 February 1983)

\begin{abstract}
Early growth of the filamentous actinomycete Streptomyces coelicolor A3(2) on solid medium was investigated. Growth kinetics were similar to those of filamentous fungi in that total mycelial length and the number of branches increased exponentially at the same specific rate, with attainment of a constant hyphal growth unit. However, both germ tube and branch hyphae showed initial linear, rather than exponential extension. Logarithmic relationships were found between branch order and both branch length and branch number, although the constancy of these relationships varied during growth. Length ratio, obtained from branch pattern analysis, reached a constant value as did the hyphal growth unit, but branch ratio did not become constant during the period of study. There was some evidence of mycelial development analogous to that found in filamentous fungi.
\end{abstract}

\section{INTRODUCTION}

Filamentous actinomycetes form circular colonies when grown on solid medium, suggesting that they have a regulation of growth and branching similar to that of filamentous fungi. Growth kinetics of filamentous fungi on solid media are characterized by an exponential increase in the total mycelial length (Trinci, 1978). This is achieved by exponential branch production with individual branches extending at a linear rate, after a short exponential growth phase (Zalokar, 1959; Trinci, 1969). The concept of the hyphal growth unit (Plomley, 1959; Trinci, 1974), representing the mean cytoplasmic volume supporting apical growth, is now well established in many filamentous fungi (Trinci, 1978) and has led to proposals on control of branch initiation (Katz et al., 1972). Tip growth of streptomycetes was first reported by Gottlieb (1953) and has been demonstrated by autoradiography in Streptomyces antibioticus (Brana et al., 1982). Hyphal growth has also been studied in Streptomyces hygroscopicus (Schuhmann \& Bergter, 1976) and 'Streptomyces granaticolor' (Kretschmer, 1982) and in both cases a constant hyphal growth unit results from similar specific rates of increase in the total mycelial length and the number of hyphal tips.

The morphology of many branching systems has been investigated using the method of Strahler (1957). In this method branches are sequentially ordered according to the position of their parental hyphae. Thus, first-order branches subtend no branches, second-order branches only first-order branches, third-order branches only second-order branches and so on. The length of a second-order branch is the distance from its origin to the apex of the longest firstorder branch which it subtends; the lengths of higher order branches are treated similarly. Plots of the average branch length and the number of branches as a function of branch order have been found to increase exponentially for a wide range of systems (Prosser, 1979). The length and branch ratio are calculated as the absolute values of the antilogs of these respective plots and are useful in comparing systems of different scale. 
Similar ratios have been found for the many different systems analysed, for example branch ratios of 3.5 and 3.2 and length ratios of 2.3 and 2.4 were found for river drainage basins and the root systems of trees respectively (Leopold, 1971). Although a logarithmic relationship for the branch ratio is found in all root systems studied, variation in values is seen between and within families (Fitter, 1982). In microbial systems the ratios of both Thamnidium elegans (Gull, 1975) and Candida albicans (Gow \& Gooday, 1982a) have been found to fall within similar ranges. The logarithmic relationships between branch number and length and branch order represent the most efficient method for a branching system to reach maximum surface area with the minimum length of filament. It is therefore of importance to establish their existence in actinomycetes. The significance of actual values for ratios is less clear, and these ratios were investigated here for their possible use as comparative descriptive parameters.

This paper describes the early mycelial growth and branching of Streptomyces coelicolor A3(2) on solid media. Branch pattern analysis was investigated with emphasis on variation of branch and length ratios with colony development. The significance of these results is discussed in relation to growth of other filamentous micro-organisms.

\section{METHODS}

Organism and spore harvest. Streptomyces coelicolor A3(2), maintained in the culture collection of the John Innes Institute, Colney Lane, Norwich, U.K., was kindly supplied by Professor D. A. Hopwood. A frozen stock spore suspension used throughout the experiments was obtained by the following method, which combined techniques of Hirsch \& Ensign (1976) and Wellington \& Williams (1978). Oatmeal agar plates (Shirling \& Gottlieb, 1966) were inoculated by spreading and incubated at $30^{\circ} \mathrm{C}$ for $10 \mathrm{~d}$, by which time there was dense sporulation. Spores were dislodged by gently rolling approximately 200 sterile acid-washed glass beads ( 2 to $4 \mathrm{~mm}$ diameter) over the surface of the colonies. The beads were transferred to cold $0.05 \mathrm{M}$-Tris/HCl buffer $(\mathrm{pH} 7 \cdot 2)$, and vigorously shaken to remove the spores. The resultant spore suspension was decanted and vortexed for about 2 min to separate spore aggregates. The suspension was filtered through cotton wool to remove mycelial debris and washed three times with Tris buffer containing $0.01 \%(\mathrm{v} / \mathrm{v})$ Tween 80 by centrifuging at 2000 r.p.m. for about 5 min. After the final washing, the pellet was resuspended in $10 \%(\mathrm{v} / \mathrm{v})$ glycerol and stored at $-20^{\circ} \mathrm{C}$. The resultant stock spore suspension remained viable (despite many freeze-thaw cycles) and showed no significant loss in the rate or extent of germination.

Growth medium and slide culture technique. Growth experiments were carried out on minimal medium (MM), (Hopwood, 1960) containing glucose $\left(0.4 \mathrm{~g} \mathrm{l}^{-1}\right)$ and Oxoid agar no. $3(1.5 \%, \mathrm{w} / \mathrm{v})$. The depth of the medium was kept constant at $2.75 \mathrm{~mm}$. Spore suspensions were prepared by serial dilution of the frozen stock in Tris buffer $\mathrm{pH} 7.2$ to give about 200 colonies per plate and were spread on to MM agar overlaid with a cellophane membrane. Cellophane membranes (Philip-Harris Ltd) were boiled for $30 \mathrm{~min}$ in distilled water to remove any plasticizers. Sterilization between layers of moist filter paper prevented crinkling and consequent uneven attachment to the agar surface. The membranes allow water and nutrient diffusion to the growing colony but prevent the hyphae from penetrating the agar. Plates were incubated at $30^{\circ} \mathrm{C}$ for 5 to $8 \mathrm{~h}$ and examined for germ tube outgrowth. A slab of agar (about $4 \times 2 \mathrm{~cm}$ ) with its cellophane overlay was then transferred into a glass-based plastic box and placed on the microscope stage. The microscope was enclosed within a Perspex box maintained at $30^{\circ} \mathrm{C}$. Dehydration of the agar block and crinkling of the cellophane membrane were prevented by placing moist filter paper over the edges of the block and maintaining a high humidity. The microscope light was switched off when not in use. Germ tube outgrowth and branch formation were followed using time-lapse photography. Colonies with thirdand fourth-order branches were photographed for branch pattern analysis.

Analysis of data. Negatives of the growing colony were projected on to a white board and the extending hyphae drawn at each time interval. Measurements of the mycelial 'tree' were obtained from diagrams of the final colony using a MOP 10 digital image analyser (Reichert) and Hewlett Packard HP85 minicomputer. The final enlargement size was calculated from negatives of a micrometer slide and was entered directly into the computer program. This program facilitated analysis of the colony, and the following output was obtained at each time interval: (1) length $(\mu \mathrm{m})$ of each hypha; (2) number of branches; (3) parental branch number of any newly formed hyphae and their points of origin; (4) total length of mycelium $(\mu \mathrm{m})$; (5) the hyphal growth unit [total mycelial length $(\mu \mathrm{m}) /$ number of branches]; (6) the branch and length ratio calculated according to Strahler (1957).

The distance between the hyphal apex and the first branch and distance between neighbouring branches were also measured using the same method, as were measurements required for branch pattern analysis. Rates of extension of individual hyphae from ten mycelial trees were calculated by linear regression analysis of the increase in individual hyphal length vs. time on both linear and logarithmic plots. Rates of increase of total mycelial length and number of branches were also calculated. Two methods were used to classify branches. For analysis of initial 
growth kinetics, hyphae that arise directly from the germ tube are termed primary branches, while hyphae arising from primary branches are termed secondary branches, etc. For branch pattern analysis, branches were sequentially ordered according to the method of Strahler (1957) described above. All measurements are given as mean values \pm standard error.

\section{RESULTS}

Extension of a typical germ tube, plotted on logarithmic and linear scales, is shown in Fig. 1. There was a 5 to $10 \mathrm{~h}$ period prior to germ tube outgrowth during which the spores became swollen and lost their refractility. The kinetics of germ tube extension were studied in 26 germ tubes, and all attained a linear extension rate which was sometimes preceded by a slower rate. Extension rates of hyphae of several colonies with no tertiary branches and a single large colony with many tertiary branches are given in Table 1 . All branches grew at a linear rate with primary branches generally exhibiting an initial slow period of extension which was less evident in branches of higher order. In the smaller colonies, germ tube extension rate was greater than those of primary and secondary branches at the $10 \%$ level, but not the $5 \%$ level of significance. In the larger colony, all hyphae extended at equal rates (no difference at the $5 \%$ level of significance), and all extension rates were greater than those in the younger colonies. The development of colonies of $S$. coelicolor usually involved the outgrowth of more than one germ tube from a single spore (a maximum of four germ tubes was observed). Often a new germ tube formed after other germ tubes had developed into colonies with tertiary branches. The establishment of a circular colony with hyphae radiating outwards from the spore indicates that growth is polarized and that hyphal interactions may occur. Visual observations revealed that branches often grew alongside each other for distances up to $15 \mu \mathrm{m}$ before diverging; hyphae only occasionally crossed over each other. In some mycelia, a branch would extend such that its apex almost touched another branch. On these occasions growth of that tip would stop and branch

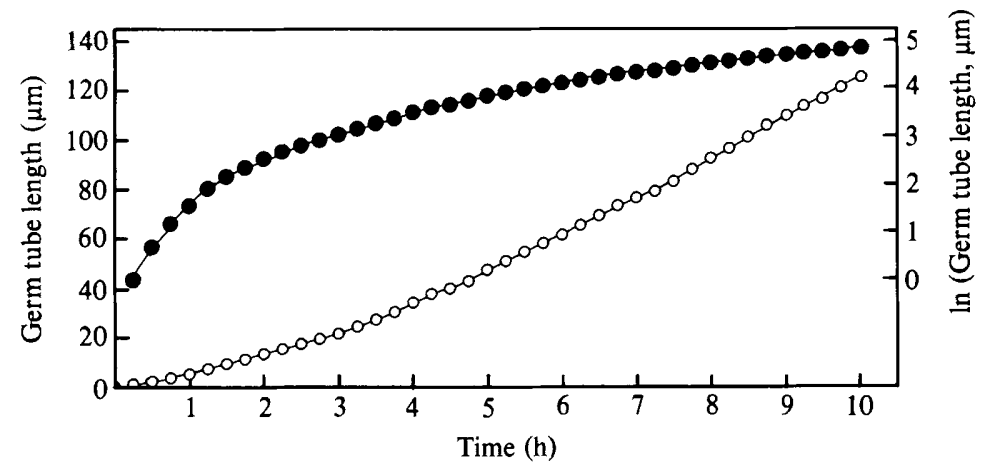

Fig. 1. Increase in $S$. coelicolor germ tube length as a function of time plotted on linear $(O)$ and logarithmic (O) scales.

Table 1. Hyphal extension rates for $S$. coelicolor grown on minimal medium from young colonies and (in parentheses) a single large colony

The values are given as mean \pm standard error for the number of replicates stated.

$\begin{array}{lcc}\text { Branch order } & \begin{array}{c}\text { No. of } \\ \text { replicates }\end{array} & \begin{array}{c}\text { Extension rate } \\ \left(\mu \mathrm{m} \mathrm{h}^{-1}\right)\end{array} \\ \text { Germ tube } & 10(3) & 13 \cdot 1 \pm 0 \cdot 9(17 \cdot 5 \pm 0 \cdot 3) \\ \text { Primary } & 20(10) & 10 \cdot 8 \pm 0 \cdot 7(16 \cdot 2 \pm 0 \cdot 9) \\ \text { Secondary } & 20(10) & 10 \cdot 8 \pm 0 \cdot 6(17 \cdot 8 \pm 1 \cdot 4) \\ \text { Tertiary } & \text { ND (10) } & \text { ND }(17.5 \pm 1 \cdot 1)\end{array}$

ND, Not determined. 


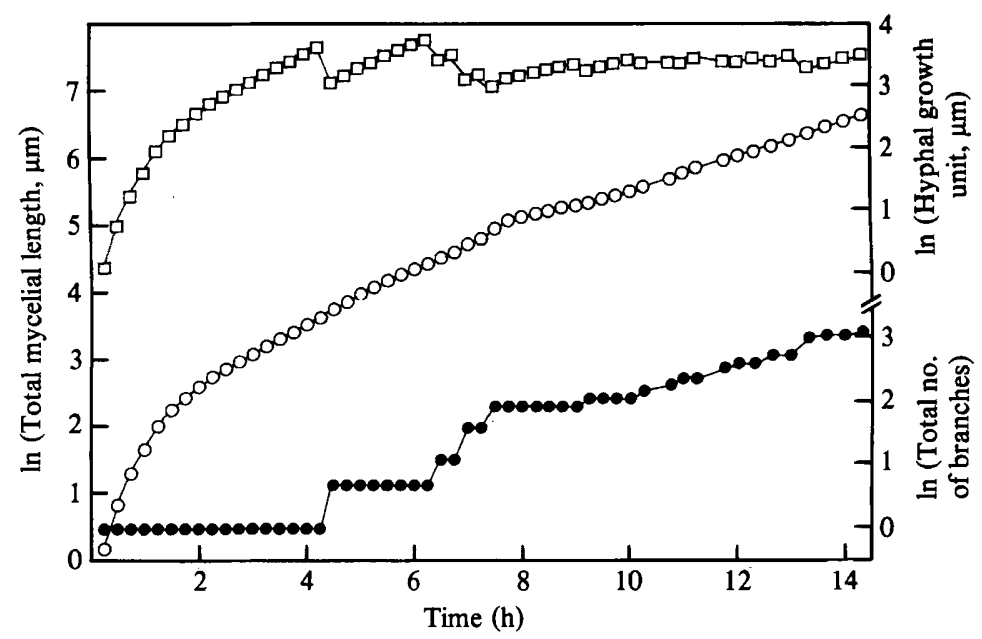

Fig. 2. Total mycelial length (O), number of tips (O) and length of the hyphal growth unit $(\square)$ in a developing mycelium of $S$. coelicolor on minimal medium.

initiation would occur at a site abnormally close to the apex. Cessation of growth also resulted from growth into crowded areas. The proportions of branches which stopped growing in the large colony were $47 \%, 60 \%$ and $44 \%$ for primary, secondary and tertiary branches, respectively.

Figure 2 represents the development of one mycelial tree within the large colony described above, which produced three germ tubes. After a period of discontinuous tip production, the total number of branches increased at an exponential rate similar to that for the increase in total mycelial length. The initial non-exponential increase in total mycelial length preceded the phase of continuous branch production and resulted from linear extension of individual hyphae. Subsequently, individual hyphae extended at a linear rate while the total mycelial length increased exponentially due to branches being initiated at an exponential rate. The specific rates of increase in total mycelial length and total number of branches were $0.26 \pm 0.002$ and $0.25 \pm$ 0.001 respectively. The hyphal growth unit initially showed damped oscillations and subsequently attained a constant value. The mean hyphal growth unit for 12 mycelial trees was $32.63 \pm 1.60 \mu \mathrm{m}$.

Distances between the hyphal apex and the first branch were normally distributed (Fig. $3 a$ ). The mean distance for 150 samples was $35.2 \pm 1.12 \mu \mathrm{m}$. Distances between neighbouring branches (Fig. $3 b$ ) exhibited a log normal distribution with a mean distance for 200 samples of $9.91 \pm 0.52 \mu \mathrm{m}$, which is higher than the modal value of $6.26 \mu \mathrm{m}$, suggesting branch formation behind the most proximal lateral branch.

Branch pattern analysis of several colonies showed logarithmic relationships between both the number of branches and mean branch length as a function of branch order (Fig. 4). The length and branch ratios of 11 third-order mycelia had mean values of $2.29 \pm 0.25$ and $3.58 \pm$ 0.32 respectively. The length and branch ratios of the fourth-order branching pattern analysed in Fig. 4 are lower than those of the third-order system. This is expected, as both ratios are dependent on the extent of branch development (Fig. 5). This graph shows that branch ratio increases in a stepwise fashion, each increase occurring when a new branch of the same order is formed. As a consequence the length ratio decreases when the number of branches remains constant and increases with the initiation of a new branch. Similarly, both ratios decrease sharply when a branch of higher order is produced. The variation in average length of a particular branch order as a function of time (for the mycelial tree analysed in Fig. 5) is shown in Fig. 6. The mean length of a given branch order initially increases at a linear rate. When a new branch is formed, the mean branch order length is approximately halved. Thus, values for the mean length of a particular branch order show damped oscillations and it can be predicted that a 

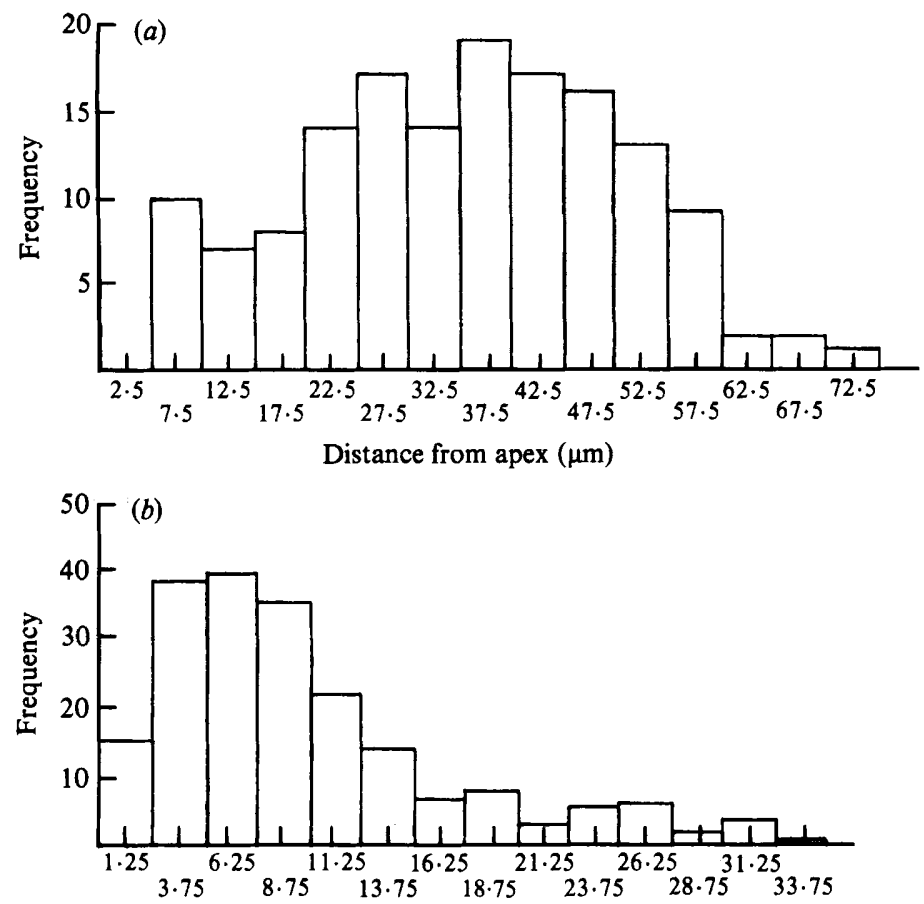

Distance between neighbouring branches $(\mu \mathrm{m})$

Fig. 3. Frequency distribution of distances between (a) the hyphal apex and the first branch and $(b)$ neighbouring branches of $S$. coelicolor.
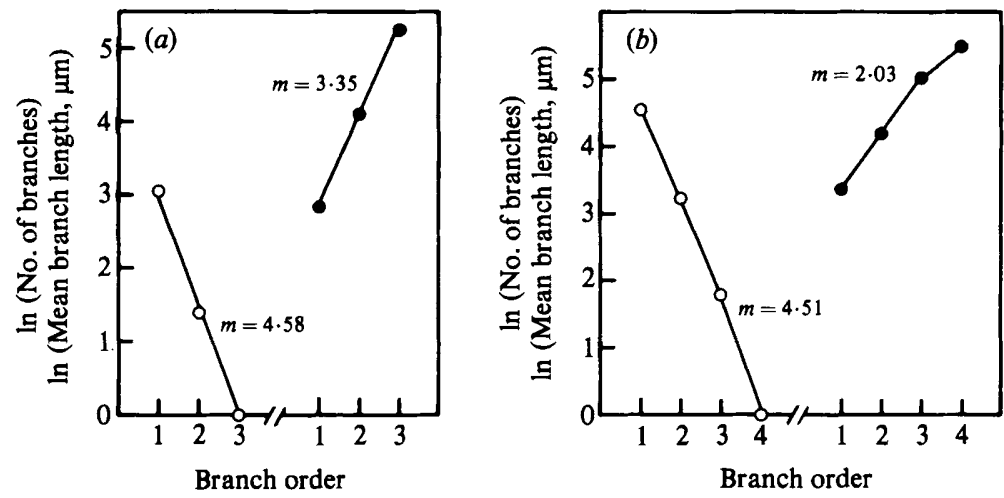

Fig. 4. Plots of the natural logarithm of the number of branches $(O)$ and the mean branch length $(O)$ of given branch order of $(a)$ a third-order and $(b)$ a fourth-order branch system of $S$. coelicolor. The absolute values of the antilog of the gradients $(m)$ of these lines indicate the branch and length ratios respectively.

constant mean branch order length will eventually be achieved, as is in fact seen for first-order branches (Fig. 6). Branch and length ratios would subsequently be expected to approach constant values. Thus, in Fig. 5 the length ratio has become constant whereas the branch ratio still shows oscillations which decrease as a higher branch order is achieved. The branch ratio will require longer to reach a constant value because of the nature of the sequential branch order analysis. 


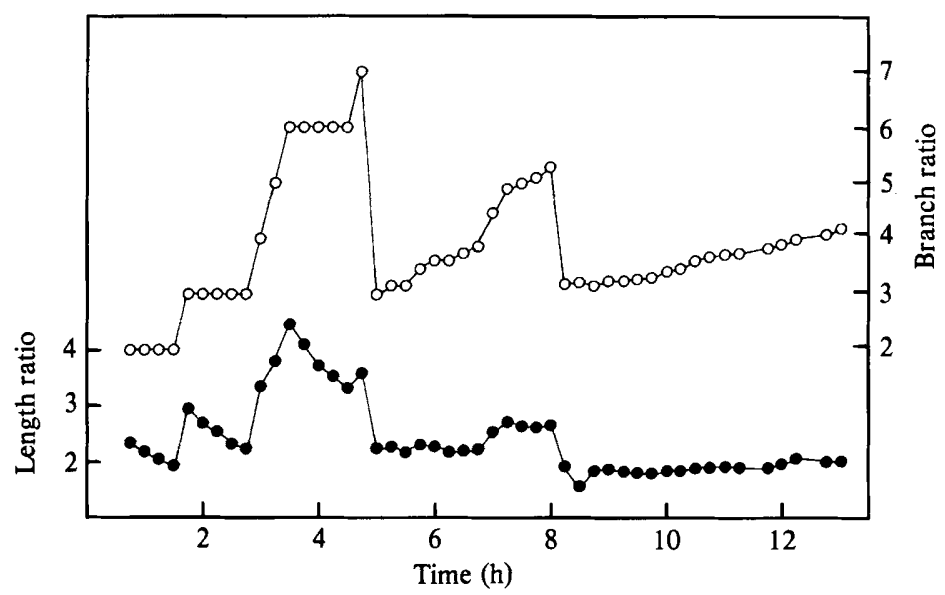

Fig. 5. Variation in the branch ratio $(O)$ and length ratio (O) of a fourth-order mycelium of $S$. coelicolor as a function of time.

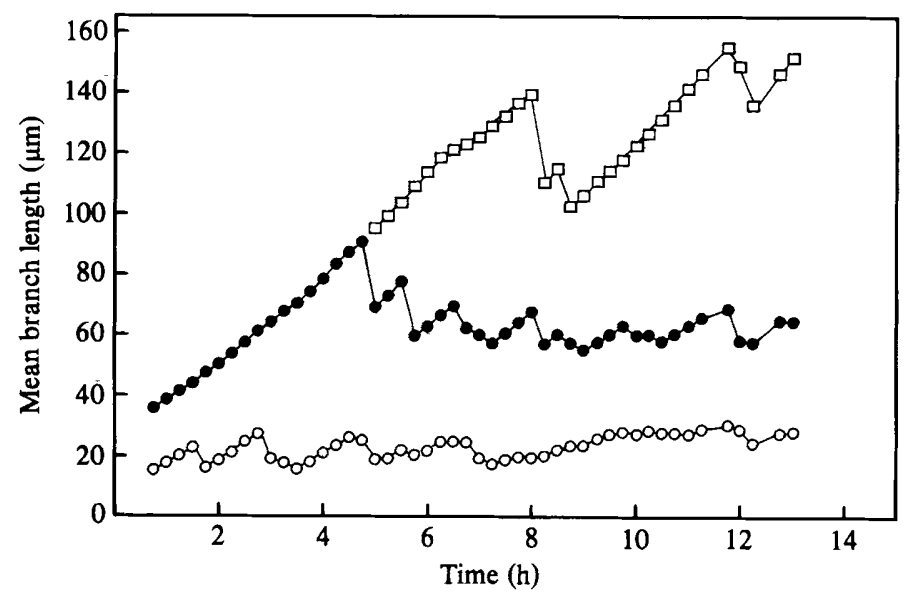

Fig. 6. Variation in mean length of first-order $(O)$, second-order $(\bigcirc)$ and third-order $(\square)$ branches in a developing mycelium of $S$. coelicolor grown on minimal medium.

\section{DISCUSSION}

Growth and development of $S$. coelicolor colonies on solid media show many similarities to those of filamentous fungi. After initial development, both the total mycelial length and number of branches increase at similar exponential rates with the consequent attainment of a constant hyphal growth unit. Schuhmann \& Bergter (1976) and Kretschmer (1982) both reported exponential extension, for germ tubes of $S$. hygroscopicus and ' $S$. granaticolor' respectively. However, the germ tubes of $S$. coelicolor, unlike the majority of filamentous fungi studied (Trinci, 1971), initially extend at a linear rather than an exponential rate. Linear extension of germ tubes has also been reported for the fungus Chaetomium globosum (Plomley, 1959) and for the dimorphic yeast Candida albicans (Gow \& Gooday, 1982a). In the latter case, this is thought to result from extensive vacuolation in the parental yeast cell and sub-apical regions of hyphae (Gow \& Gooday, 1982b). Branches of $S$. coelicolor also extended at a linear rate from their initiation, showing another deviation from standard fungal growth kinetics (Trinci, 1970). This linear branch extension suggests that the linear growth of germ tubes does not result from any physical disturbance encountered during preparation and transfer of the slide culture to the microscope chamber. There is some evidence that germ tube hyphae extend faster than branches they 
subtend in young colonies, but any initial advantage that they have is subsequently lost, with all hyphae extending at the same rate. The faster extension rates found in the larger colony (Table 1) may result from statistical variation between different colonies. Alternatively, it may involve a form of mycelial development. In filamentous fungi, such development is termed differentiation (Steele \& Trinci, 1975), but this involves differences in extension rates between branches of different order, which are not found here. Such a process may occur in actinomycetes but may require analysis of colonies at a later stage of growth.

Although it has never been systematically studied, there appears to be a correlation between hyphal diameter and extension rate in filamentous fungi. This relationship can be extended to dimorphic yeasts and filamentous actinomycetes. Candida albicans has a primary branch extension rate of $18.9 \mu \mathrm{m} \mathrm{h}^{-1}$ compared to $11.2 \mu \mathrm{m} \mathrm{h}^{-1}$ for $S$. coelicolor. The respective hyphal diameters are $2.40 \mu \mathrm{m}$ and $1.02 \mu \mathrm{m}$. The attainment of a constant hyphal growth unit suggests mechanisms of branch initiation similar to those of filamentous fungi (Katz et al., 1972). Although apical extension has been reported frequently for streptomycetes, there has been no indication as to the precise cellular mechanism. It is not yet possible to explain branch initiation and development in terms of cellular events as in filamentous fungi (Prosser \& Trinci, 1979).

Sub-apical branching enables continuation of autocatalytic growth in filamentous organisms when individual hyphae extend at a linear rate. Biosynthesis in hyphal regions distant from the apex results in formation of a new apex. However, towards the centre of a growing colony conditions will become less favourable, because of substrate depletion, accumulation of secondary metabolites and space limitation. The final interbranch distance in these regions will therefore be reached when growth ceases. In addition, work on other streptomycete strains suggests an analogy with the cell cycle of unicellular bacteria and a minimum interbranch and intercalary compartment length, equivalent to the unit cell length $(1.39 \mu \mathrm{m})$ of Escherichia coli (Donachie et al., 1976). Szesák et al. (1973) found that hyphae of Streptomyces griseus, fragmented by chemical and physical methods, had a size similar to this unit cell length. Riesenberg et al. (1979) found that the distance between neighbouring branches of $S$. hygroscopicus was equivalent to the prokaryote cell unit size. Kretschmer (1982) classified cells of $S$. granaticolor into groups with different interseptal distances, the smallest ranging from 3 to $9 \mu \mathrm{m}$. A model for growth was proposed with a maximum of one branch arising from each intercalary compartment. The mean and modal values for distances between neighbouring branches of $S$. coelicolor $(9.91$ and $6.75 \mu \mathrm{m})$ exceed the minima in these studies and also the $E$. coli unit cell length. This may, however, arise through measurement of young colonies where growth at the centre had not ceased and branching had not reached its maximum.

The deviation from linear relationships between branch number and branch length as a function of branch order was frequently observed. This is most apparent when a new branch order is initiated. Subsequent formation of more branches of this higher order, and extension and formation of branches of lower orders, restores the linear relationship. In addition, branch ratio did not reach a constant value and length ratios became constant only after a period of fluctuation similar to that for the hyphal growth unit. In comparing ratios from different systems, it must be ensured that this constant region has been attained. Calculation of ratios is particularly difficult for dense mycelia and mean branch length is a more reliable descriptive parameter.

We would like to thank Dr S. T. Williams and Dr G. W. Gooday for useful advice and comments. E.J.A. acknowledges receipt of a NERC Research Studentship.

\section{REFERENCES}

Brana, A. F., Manzanal, M.-B. \& Hardisson, C. (1982). Mode of cell wall growth of Streptomyces antibioticus. FEMS Microbiology Letters 13, 231-235.

Donachie, W. O., BegG, K. J. \& Vicente, M. (1976). Cell length, cell growth and cell division. Nature, London 264, 328-333.
FITTER, A. H. (1982). Morphometric analysis of root systems: application of the technique and influence of soil fertility on root system development in two herbaceous species. Plant, Cell and Environment 5, 313-322.

GotTliEB, D. (1953). The physiology of the Actinomy- 
cetes. In Actinomycetales, Morphology, Biology and Systematics. Sixth International Congress for Microbiology, pp. 122-136. Rome: Fondazione Emanuele Paterno.

Gow, N. A. R. \& Gooday, G. W. (1982a). Growth kinetics and morphology of colonies of the filamentous form of Candida albicans. Journal of General Microbiology 128, 2187-2194.

Gow, N. A. R. \& GoOdAY, G. W. (1982b). Vacuolation, branch production and linear growth of germ tubes of Candida albicans. Journal of General Microbiology 128, 2195-2198.

GuLL, K. (1975). Mycelial branch patterns of Thamnidium elegans. Transactions of the British Mycological Society 64, 321-363.

Hirsch, C. F. \& Ensign, J. G. (1976). Nutritionally defined conditions for germination of Streptomyces viridochromogenes spores. Journal of Bacterialogy 126 , 13-23.

HoPwOOD, D. A. (1960). Phase contrast observations on Streptomyces coelicolor. Journal of General Microbiology 22, 295-302.

Katz, D., Goldstein, D. \& Rosenberger, R. F. (1972). Model for branch initiation in Aspergillus nidulans based on measurements of growth parameters. Journal of Bacteriology 109, 1097-1100.

KRETSCHMER, S. (1982). Dependence of the mycelial growth pattern on the individually regulated cell cycle in Streptomyces granaticolor. Zeitschrift für allgemeine Mikrobiologie 22, 335-347.

LeOPOLD, L. B. (1971). Trees and streams: the efficiency of branching patterns. Journal of Theoretical Biology 31, 339-354.

Plomley, N. J. B. (1959). Formation of the colony in the fungus Chaetomium. Australian Journal of Biological Sciences 12, 53-64.

Prosser, J. I. (1979). Mathematical modelling of mycelial growth. In Fungal Walls and Hyphal Growth, pp. 359-384. Edited by J. H. Burnett \& A. P. J. Trinci. London: Cambridge University Press.

Prosser, J. I. \& Trinci, A. P. J. (1979). A model for hyphal growth and branching. Journal of General Microbiology 111, 153-164.

RieSENBERG, D., ERDMANN, A. \& BERgTer, F. (1979)
Distribution functions of variables characterizing the mycelial morphology of Streptomyces hygroscopicus grown in glucose-limited chemostat culture. Zeitschrift für allgemeine Mikrobiologie 19, 481-487.

Schummann, E. \& Bergter, F. (1976). Mikroskopische Untersuchungen zur Wachstumskinetik von Streptomyces hygroscopicus. Zeitschrift für allgemeine Mikrobiologie 16, 201-215.

ShiRling, E. B. \& GotTlieb, D. (1966). Methods for characterization of Streptomyces species. International Journal of Systematic Bacteriology 16, 313340.

Steele, G. C. \& TRINCI, A. P. J. (1975). Morphology and growth kinetics of hyphae of differentiated and undifferentiated mycelia of Neurospora crassa. Journal of General Microbiology 91, 362-368.

STRAHLER, A. N. (1957). Quantitative analysis of watershed geomorphology. Transactions of the American Geophysical Union 38, 913-920.

Szesák, F., Skripeczky, K., Princzinger, A. \& SzABó, G. (1973). Functional units of Streptomyces hyphae equivalent with prokaryotic cells. Zentralblatt für Bakteriologie 128, 243-251.

TrincI, A. P. J. (1969). A kinetic study of the growth of Aspergillus nidulans and other fungi. Journal of General Microbiology 57, 11-24.

Trinci, A. P. J. (1970). Kinetics of apical and lateral branching in Aspergillus nidulans and Geotrichum lactis. Transactions of the British Mycological Society 55, 17-28.

TRINCI, A. P. J. (1971). Exponential growth of germ tubes of fungal spores. Journal of General Microbiology 67, 345-348.

TrincI, A. P. J. (1974). A study of the kinetics of hyphal extension and branch initiation of fungal mycelia. Journal of General Microbiology 81, 225236.

TrincI, A. P. J. (1978). Wall and hyphal growth. Science Progress 65, 75-99.

Wellington, E. M. H. \& Williams, S. T. (1978). Preservation of actinomycete inoculum in frozen glycerol. Microbios Letters 6, 151-157.

ZaLOKAR, M. (1959). Enzyme activity and cell differentiation in Neurospora. American Journal of Botany 46, 555-559. 